\title{
Streptomyces spp. from rhizosphere soil of maize with potential as plant growth promoter
}

\author{
ARIS TRI WAHYUDI ${ }^{1, \vartheta}$, JEPRI AGUNG PRIYANTO ${ }^{1}$, HANIFA NUR FIJRINA ${ }^{1}$, HIMA DEWI MARIASTUTI, \\ ABDJAD ASIH NAWANGSIH ${ }^{2}$ \\ ${ }^{1}$ Division of Microbiology, Department of Biology, Faculty of Mathematics and Natural Sciences, Institut Pertanian Bogor. Jl. Raya Dramaga, Bogor \\ 16680, West Java, Indonesia. Tel./fax.: 62-251-8622833. `email: aristri2011@gmail.com. \\ ${ }^{2}$ Department of Plant Protection, Faculty of Agriculture, Institut Pertanian Bogor. Jl. Raya Dramaga, Bogor 16680, West Java, Indonesia, West Java, \\ Indonesia
}

Manuscript received: 7 July 2019. Revision accepted: 19 August 2019.

\begin{abstract}
Wahyudi AT, Priyanto JA, Fijrina HN, Mariastuti HD, Nawangsih AA. 2019. Streptomyces spp. from rhizosphere soil of maize with potential as plant growth promoter. Biodiversitas 20:2547-2553. Actinomycete is one group of rhizobacteria that plays an important role as a plant growth promoter. This study was aimed to evaluate the potential of Actinomycetes isolated from maize rhizosphere in promoting plant growth in vitro including their ability to produce IAA, promote maize sprout growth, solubilize phosphate, and grow in $\mathrm{N}$-free medium. Thirty isolates have been isolated from maize rhizosphere using a spread plate method. All 30 isolates were probably not pathogenic to plants as tested by hypersensitivity reaction test on tobacco leaves. Based on the colorimetric assay, 30 isolates (100\%) were able to produce IAA with concentrations ranging from 1.05 to $26.89 \mathrm{ppm}$. The highest concentration of IAA $(26.89 \mathrm{ppm})$ produced by ARJ 21 and the lowest IAA concentration $(1.05 \mathrm{ppm})$ produced by ARJ 12 . By using the Ragdoll method, it showed that 9 isolates $(30 \%)$ were able to promote maize sprout growth significantly on five growth parameters including primary root length, stem length, number of lateral roots, wet weight and dry weight. Twenty-one isolates (70\%) were capable of solubilizing phosphate in Pikovskaya medium containing tricalcium phosphate. Also, 30 isolates (100\%) were able to grow on N-free medium, suggesting their ability to fix nitrogen. Based on 16S-rRNA, five potential isolates with plant growth-promoting properties were highly similar to Streptomyces spp. Based on their potential characters, these Actinomycetes isolates have the potential to be further developed as a biofertilizer agent for sustainable maize farming.
\end{abstract}

Keywords: IAA, nitrogen fixation, phosphate solubilization, plant growth, rhizosphere Actinomycetes

\section{INTRODUCTION}

Maize (Zea mays L) is one of the important food sources of carbohydrates, besides wheat, sorghum, and rice. As a source of carbohydrates, some people consume it as a staple food. Maize is also used as a source of vegetable oil, cornstarch, ethanol, organic acids, and animal feed. Maize farming currently depends on the use of chemical/ inorganic fertilizers. Kresnatita et al. (2013) reported that the use of inorganic fertilizers increased the growth and yield of sweet corn plants, by $114.8 \%$ compared to the treatment of organic fertilizers. However, the use of inorganic fertilizers extensively in agricultural cultivation will adversely affect the environment such as increased soil salinity, accumulation of heavy metals, eutrophication, and accumulation of nitrates in waters (Savci 2012). Therefore, other alternative fertilizers which environmentally friendly are needed for sustainable maize farming.

Plant growth-promoting rhizobacteria (PGPR) is currently being the main topics of research for developing biofertilizer agents. Some beneficial microorganisms isolated from soil have the potential to be used as an alternative to inorganic fertilizers, especially rhizobacteria. Rhizobacteria are found around plant roots and can promote plant growth. PGPR could enhance plant growth through direct and indirect mechanisms. On the direct mechanism, PGPR is capable of producing phytohormones, fixing nitrogen, and solubilizing phosphate (Marques et al. 2010). Whereas in the indirect mechanism, PGPR increases plant growth by secreting phytopathogenic inhibitors, cell wall degrading enzymes, and siderophore (Sreevidya 2016). The exploration of indigenous Actinomycetes strains may lead to potential biological agents that are suitable for local conditions to enhance sustainable maize production.

Among all bacterial groups, Actinomycetes, a filamentous Gram-positive bacteria, have some potential characters which could stimulate plant growth by producing Indole Acetic Acid (Jeon et al. 2003), siderophore and solubilizing phosphates (Patil et al. 2010). Streptomyces, one of the actinomycetes genus, have been considered as the most promising group for improving plant growth, soil fertility, and increasing plant biomass (Viaene et al. 2016). Isolation and screening of Actinomycetes isolated from maize rhizosphere were expected to obtain some promising isolates attributed to plant growth-promoting traits. In this present study, research focused on the isolation and screening of maize rhizosphere Actinomycetes as a plant growth promoter in vitro to obtain ideal candidates for developing natural fertilizer. 


\section{MATERIALS AND METHODS}

\section{Isolation of maize rhizosphere Actinomycetes}

Maize rhizosphere Actinomycetes were isolated by using serial dilution method. The soil used in this study was collected from maize plantations in East Nusa Tenggara, Indonesia. The selective medium, Humic Acid Vitamin Agar (HVA; composition: $\mathrm{CaCO}_{3} 0.020 \mathrm{~g}, \mathrm{KCl}$ $1.7 \mathrm{~g}$, humic acid $1 \mathrm{~g}, \mathrm{Na}_{2} \mathrm{HPO}_{4} 0.5 \mathrm{~g}, \mathrm{MgSO}_{4} .7 \mathrm{H}_{2} \mathrm{O} 0.05$ g, $\mathrm{Na}_{2} \mathrm{HPO}_{4} 0.5 \mathrm{~g}, \mathrm{FeSO}_{4} .7 \mathrm{H}_{2} \mathrm{O} 0.01 \mathrm{~g}, \mathrm{~B}$-vitamins $5 \mathrm{~mL}$, cycloheximide $0.05 \mathrm{~g}$, nalidixic acid $0.02 \mathrm{~g}$, agar $15 \mathrm{~g}$, distilled water $1000 \mathrm{~mL}, \mathrm{pH}$ 7.2) was used. Prior serial dilution, one $\mathrm{g}$ of soil was preheated at $60^{\circ} \mathrm{C}$ for $15 \mathrm{~min}$, then homogenized in $9 \mathrm{~mL}$ of $0.85 \% \mathrm{NaCl}$ solution $\left(10^{-1}\right)$ and further used for subsequent dilution $10^{-6}$. Nearly 100 $\mu \mathrm{L}$ of last three dilutions were spread on top of HVA medium and were incubated for 14 days at room temperature $\left( \pm 27^{\circ} \mathrm{C}\right)$. The growing Actinomycetes colonies were then purified in the International Streptomyces Project medium no.4 (ISP2; composition: soluble starch $10 \mathrm{~g}$, $\mathrm{MgSO}_{4} 1 \mathrm{~g}, \mathrm{~K}_{2} \mathrm{HPO}_{4} 1 \mathrm{~g}, \mathrm{NaCl} 1 \mathrm{~g},\left(\mathrm{NH}_{4}\right)_{2} \mathrm{SO}_{4} 2 \mathrm{~g}, \mathrm{CaCO}_{3}$ $0.001 \mathrm{~g}, \mathrm{MnCl}_{2} 0.001 \mathrm{~g}, \mathrm{ZnSO}_{4} 0.001 \mathrm{~g}, \mathrm{FeSO}_{4} 0.001 \mathrm{~g}$, distilled water $1000 \mathrm{~mL}, \mathrm{pH} 7.2$ ).

\section{Hypersensitivity reaction test}

Thirty Actinomycetes isolates were tested to determine their pathogenicity to plants by using tobacco leaves. One $\mathrm{mL}$ of seven days-old Actinomycetes culture was injected into the interveinal tissue using a sterile syringe (without needles) on the abaxial surface of tobacco leaves. The necrotic area in the tissue formed after 24-48 hours of injection indicates a positive hypersensitivity reaction (Wiraswati et al. 2019). Sterile distilled water and Xanthomonas oryzae pv. oryzae (Xoo) were used as a negative and positive control, respectively.

\section{Measurement of indole-acetic acid (IAA) production}

IAA production of each Actinomycetes isolate was determined by using the colorimetric method (Gopalakrishnan et al. 2014). Two plugs of inoculums ( \pm 0.8 diameter) were inoculated in $20 \mathrm{~mL}$ of ISP2 liquid medium supplemented with $0.2 \mathrm{~mL}$ of $0.2 \%$ L-tryptophan and incubated in an agitated incubator at $120 \mathrm{rpm}$ at room temperature $\left( \pm 27^{\circ} \mathrm{C}\right)$ for 10 days. After incubation completed, one $\mathrm{mL}$ supernatant of each culture was mixed with $4 \mathrm{~mL}$ of Salkowski reagent $\left(\mathrm{FeCl}_{3} \cdot 6 \mathrm{H}_{2} \mathrm{O} \quad 0.05 \% 7.5\right.$ $\mathrm{mL}, \mathrm{H}_{2} \mathrm{SO}_{4} 150 \mathrm{~mL}$, distilled water $250 \mathrm{~mL}$ ). The homogenized solutions were then incubated in the dark for about $30 \mathrm{~min}$. IAA concentration was calculated based on the standard curve. Staphylococcus aureus IPBCC and Pseudomonas aeruginosa IPBCC (Bogor Agricultural University Culture Collection-Indonesia) were used as a negative and positive control, respectively. Each sample was tested in Duplo.

\section{Screening of plant growth-promoting (PGP) activities in vitro}

PGP properties of each Actinomycete isolate were evaluated by Ragdoll method as described by Sreevidya et al. (2016). Before use, three plugs of Actinomycetes inoculum were inoculated in $50 \mathrm{~mL}$ of ISP4 medium and then incubated in a shaker $(120 \mathrm{rpm})$ at room temperature $\left( \pm 27^{\circ} \mathrm{C}\right)$ for 7 days. The surface of maize seeds was sterilized by using $96 \%$ ethanol for $10 \mathrm{~s}$, and $3 \%$ of $\mathrm{NaOCl}$ for 10 min, 6 times washed with sterile distilled water. The sterilized seeds were then soaked in sterilized distilled water for $24 \mathrm{~h}$. The submerged seeds were selected for cultivation in sterile wet tissues and incubated for $24 \mathrm{~h}$. The germinated seeds with a radicular length of $2-3 \mathrm{~mm}$ were selected for further testing. The germinated seeds were then soaked in Actinomycetes culture containing individual strain for $60 \mathrm{~min}$. The soaked germinated seeds were cultivated on a wet paper towel, folded and rolled and incubated at $\pm 27^{\circ} \mathrm{C}$ for 5 days. The seeds treated with sterile distilled water and uninoculated ISP4 medium were served as control. After the incubation period, five parameters, including the number of lateral roots, hypocotyl length, radicular length, dry weight, and wet weight, were observed. This assay was conducted in triplicates, and each replication consists of nine sprouts. The selected Actinomycetes isolates were grouped into 3 experimental groups based on the time of the test conducted. The data were analyzed by One Way Analysis of Variance (ANOVA), and the significant differences among treatments were tested by Duncan Multiple Range Test $(\alpha=0.05)$.

\section{Growth of Actinomycetes in $\mathbf{N}$-free medium}

Each Actinomycete isolates grown by streaking on $\mathrm{N}$ free medium agar and were incubated for 7 days at room temperature $\left( \pm 27^{\circ} \mathrm{C}\right)$. $\mathrm{N}$-free medium contained $(\mathrm{g} / \mathrm{L})$ : dextrose (5), $\mathrm{MgSO}_{4}(0.065), \mathrm{KH}_{2} \mathrm{PO}_{4}(3), \mathrm{K}_{2} \mathrm{HPO}_{4}$ (1), $\mathrm{CaCl}_{2} .2 \mathrm{H}_{2} \mathrm{O}(0.07), \mathrm{CoCl}_{2} \cdot 6 \mathrm{H}_{2} \mathrm{O}\left(1.20 \times 10^{-4}\right), \mathrm{FeCl}_{3} \cdot 6 \mathrm{H}_{2} \mathrm{O}$ $(0.01), \quad \mathrm{H}_{3} \mathrm{BO}_{4} \quad\left(3 \times 10^{-6}\right), \quad \mathrm{ZnSO}_{4} .7 \mathrm{H}_{2} \mathrm{O} \quad\left(2.90 \times 10^{-4}\right)$, $\mathrm{Na}_{2} \mathrm{MoO}_{4} .2 \mathrm{H}_{2} \mathrm{O} \quad\left(240 \times 10^{-6}\right), \quad \mathrm{MnSO}_{4} \cdot \mathrm{H}_{2} \mathrm{O} \quad\left(1.83 \times 10^{-6}\right)$, $\mathrm{CuSO}_{4} .5 \mathrm{H}_{2} \mathrm{O}\left(1.30 \times 10^{-4}\right)$, agar (15). Escherichia coli strain DH5 $\alpha$ (Collection of Microbiology Laboratory, Bogor Agricultural University) was used as a negative control.

\section{Phosphate solubilization test}

Phosphate solubilizing activity was tested by inoculating one plug of Actinomycetes colony onto the surface of Pikovskaya medium and incubated at $\pm 27^{\circ} \mathrm{C}$ for 7 days. The medium composition was $(\mathrm{g} / \mathrm{L}): \mathrm{NaCl}(0.2)$, $\mathrm{KCl}(0.2), \mathrm{MgSO}_{4} .7 \mathrm{H}_{2} \mathrm{O}(0.1), \mathrm{MnSO}_{4} .7 \mathrm{H}_{2} \mathrm{O}(0.0025)$, FeSO4.7 $\mathrm{H}_{2} \mathrm{O}(0.0025),\left(\mathrm{NH}_{4}\right)_{2} \mathrm{SO}_{4}(0.5)$, glucose (10), yeast extract (0.5), $\mathrm{Ca}_{3}\left(\mathrm{PO}_{4}\right)_{2}(5)$, and agar (15). Bradyrhizobium sp. (Collection of Microbiology Laboratory, Bogor Agricultural University) was served as a positive control. The clear zone around Actinomycetes colonies indicates the phosphate solubilizing activity. Phosphate solubilization index was calculated by using the following formula: (diameter of colony + diameter of the clear zone)/ diameter of colony.

\section{Identification of the potential Actinomycetes isolates}

Presto $^{\mathrm{TM}}$ Mini gDNA bacteria Kit (Geneaid) was used for extraction of genomic DNA Actinomycetes isolates as following the manufacturer's instructions. 16S-rRNA genes were amplified by using primer pair $27 \mathrm{~F}$ (5'- 
AGAGTTTGATCCTGGCTCAG-3') and 1114R (5'GAGTTGACCCCGGCRGT-3') that targeted DNA fragment approximately $1087 \mathrm{bp}$ in size (Martina et al. 2008). Fifty $\mu \mathrm{L}$ PCR mix containing: $\mathrm{MyTaq}^{\mathrm{TM}}$ HS Red Mix 2x (Bioline) $(25 \mu \mathrm{L}), 10 \mathrm{pmol} 1387 \mathrm{r}$ primers $(5 \mu \mathrm{L})$, 10 pmol $63 \mathrm{f}$ primers $(5 \mu \mathrm{L}), 100 \mathrm{ng} / \mu \mathrm{L}$ of DNA template $(4 \mu \mathrm{L})$, and adjusted with nuclease-free water $(11 \mu \mathrm{L})$ was used as PCR mix reaction. The PCR conditions were served in 30 cycles under the following condition: $94^{\circ} \mathrm{C}$ for $5 \mathrm{~min}, 94^{\circ} \mathrm{C}$ for $30 \mathrm{~s}, 55$ for $45 \mathrm{~s}, 72^{\circ} \mathrm{C}$ for $1 \mathrm{~min} 45 \mathrm{~s}$ and a final extension at $72^{\circ} \mathrm{C}$ for $10 \mathrm{~min}$. The PCR products were sequenced in First Base, Malaysia. The sequences were aligned by using the BlastN program in National Center for Biotechnology Information (NCBI) GenBank database. The 16S-rRNA sequences of each isolate were deposited to NCBI GenBank (https://www.ncbi.nlm.nih.gov). The phylogenetic tree was constructed using MEGA 7.0 software by the neighbor-joining method with 1000x bootstrap replications.

\section{RESULTS AND DISCUSSION}

\section{Actinomycetes isolated from maize rhizosphere and their hypersensitivity reaction}

Thirty isolates have successfully been isolated from maize rhizosphere by using HVA medium. These isolates were diverse in the colony and cellular morphology. All 30 isolates $(100 \%)$ and negative control (injected with sterile distilled water) showed negative hypersensitivity reaction as indicated by not-forming a necrotic area after injection of those Actinomycetes culture on tobacco leaf. Conversely, tobacco leaf injected with Xanthomonas oryzae inoculum formed a necrotic area (data not shown).

Table 1. Plant growth-promoting properties of 30 actinomycetes isolated from maize rhizosphere

\begin{tabular}{|c|c|c|c|c|c|c|c|c|}
\hline \multirow{2}{*}{ Isolate code } & \multirow{2}{*}{$\begin{array}{c}\text { IAA } \\
\text { concentration (ppm) }\end{array}$} & \multicolumn{5}{|c|}{ Growth parameters } & \multirow{2}{*}{$\begin{array}{c}\text { Growth in } N \text {-free } \\
\text { medium }\end{array}$} & \multirow{2}{*}{$\begin{array}{c}\text { Phosphate } \\
\text { solubilization index }\end{array}$} \\
\hline & & HL & $\mathbf{R L}$ & LR & WW & DW & & \\
\hline ARJ 51 & 20.74 & $\mathrm{v}$ & $\mathrm{v}$ & $\mathrm{v}$ & $\mathrm{v}$ & $\mathrm{v}$ & + & $1.53 \pm 0.05$ \\
\hline ARJ 81 & 20.64 & $\mathrm{v}$ & $\mathrm{v}$ & $\mathrm{v}$ & $\mathrm{v}$ & $\mathrm{v}$ & + & $1.89 \pm 0.25$ \\
\hline ARJ 15 & 1.76 & $\mathrm{v}$ & $\mathrm{v}$ & $\mathrm{v}$ & $\mathrm{v}$ & $\mathrm{v}$ & + & $1.33 \pm 0.05$ \\
\hline ARJ 38 & 10.19 & $\mathrm{v}$ & $\mathrm{v}$ & $\mathrm{v}$ & $\mathrm{v}$ & $\mathrm{v}$ & + & $1.40 \pm 0.02$ \\
\hline ARJ 44 & 9.07 & $\mathrm{v}$ & $\mathrm{v}$ & $\mathrm{v}$ & $\mathrm{v}$ & $\mathrm{v}$ & + & $1.42 \pm 0.03$ \\
\hline ARJ 14 & 10.37 & $\mathrm{v}$ & $\mathrm{v}$ & $\mathrm{v}$ & $\mathrm{v}$ & $\mathrm{v}$ & + & 0 \\
\hline ARJ 32 & 6.70 & $\mathrm{v}$ & $\mathrm{v}$ & $\mathrm{v}$ & $\mathrm{v}$ & $\mathrm{v}$ & + & 0 \\
\hline ARJ 16 & 6.57 & $\mathrm{v}$ & $\mathrm{v}$ & $\mathrm{v}$ & $\mathrm{v}$ & $\mathrm{v}$ & + & 0 \\
\hline ARJ 28 & 2.49 & $\mathrm{v}$ & $\mathrm{v}$ & $\mathrm{v}$ & $\mathrm{v}$ & $\mathrm{v}$ & + & 0 \\
\hline ARJ 61 & 5.74 & $\mathrm{v}$ & $\mathrm{x}$ & $\mathrm{v}$ & $\mathrm{v}$ & $\mathrm{v}$ & + & $1.32 \pm 0.15$ \\
\hline ARJ 36 & 10.64 & $\mathrm{x}$ & $\mathrm{x}$ & $\mathrm{v}$ & $\mathrm{v}$ & $\mathrm{v}$ & + & $1.43 \pm 0.14$ \\
\hline ARJ 47 & 3.02 & $\mathrm{v}$ & $\mathrm{v}$ & $\mathrm{v}$ & $\mathrm{v}$ & $\mathrm{x}$ & + & $1.48 \pm 0.04$ \\
\hline ARJ 24 & 4.26 & $\mathrm{v}$ & $\mathrm{v}$ & $\mathrm{v}$ & $\mathrm{v}$ & $\mathrm{x}$ & + & $1.45 \pm 0.16$ \\
\hline ARJ 27 & 8.41 & $\mathrm{v}$ & $\mathrm{v}$ & $\mathrm{v}$ & $\mathrm{v}$ & $\mathrm{x}$ & + & $1.14 \pm 0.08$ \\
\hline ARJ 43 & 1.67 & $\mathrm{x}$ & $\mathrm{v}$ & $\mathrm{v}$ & $\mathrm{v}$ & $\mathrm{v}$ & + & $1.45 \pm 0.07$ \\
\hline ARJ 23 & 4.01 & $\mathrm{x}$ & $\mathrm{v}$ & $\mathrm{v}$ & $\mathrm{v}$ & $\mathrm{v}$ & + & $1.40 \pm 0.26$ \\
\hline ARJ 34 & 3.01 & $\mathrm{v}$ & $\mathrm{v}$ & $\mathrm{v}$ & $\mathrm{x}$ & $\mathrm{v}$ & + & $1.44 \pm 0.04$ \\
\hline ARJ 49 & 19.26 & $\mathrm{v}$ & $\mathrm{v}$ & $\mathrm{v}$ & $\mathrm{x}$ & $\mathrm{v}$ & + & $1.09 \pm 0.08$ \\
\hline ARJ 13 & 4.88 & $\mathrm{v}$ & $\mathrm{v}$ & $\mathrm{v}$ & $\mathrm{x}$ & $\mathrm{v}$ & + & $1.49 \pm 0.04$ \\
\hline ARJ 21 & 26.89 & $\mathrm{v}$ & $\mathrm{x}$ & $\mathrm{v}$ & $\mathrm{x}$ & $\mathrm{v}$ & + & $1.50 \pm 0.024$ \\
\hline ARJ 11 & 25.80 & $\mathrm{v}$ & $\mathrm{v}$ & $\mathrm{v}$ & $\mathrm{x}$ & $\mathrm{x}$ & + & 0 \\
\hline ARJ 37 & 16.30 & $\mathrm{v}$ & $\mathrm{x}$ & $\mathrm{v}$ & $\mathrm{v}$ & $\mathrm{x}$ & + & 0 \\
\hline ARJ 22 & 15.74 & $\mathrm{x}$ & $\mathrm{v}$ & $\mathrm{v}$ & $\mathrm{v}$ & $\mathrm{v}$ & + & 0 \\
\hline ARJ 45 & 9.38 & $\mathrm{v}$ & $\mathrm{v}$ & $\mathrm{x}$ & $\mathrm{x}$ & $\mathrm{v}$ & + & $1.53 \pm 0.08$ \\
\hline ARJ 31 & 4.39 & $\mathrm{v}$ & $\mathrm{v}$ & $\mathrm{x}$ & $\mathrm{x}$ & $\mathrm{v}$ & + & $1.36 \pm 0.06$ \\
\hline ARJ 48 & 15.12 & $\mathrm{x}$ & $\mathrm{v}$ & $\mathrm{v}$ & $\mathrm{x}$ & $\mathrm{x}$ & + & $1.30 \pm 0.07$ \\
\hline ARJ 42 & 12.22 & $\mathrm{x}$ & $\mathrm{v}$ & $\mathrm{v}$ & $\mathrm{x}$ & $\mathrm{x}$ & + & $1.54 \pm 0.18$ \\
\hline ARJ 18 & 4.75 & $\mathrm{x}$ & $\mathrm{x}$ & $\mathrm{v}$ & $\mathrm{x}$ & $\mathrm{x}$ & + & 0 \\
\hline ARJ 17 & 1.54 & $\mathrm{x}$ & $\mathrm{x}$ & $\mathrm{x}$ & $\mathrm{x}$ & $\mathrm{x}$ & + & 0 \\
\hline ARJ 12 & 1.05 & $\mathrm{x}$ & $\mathrm{x}$ & $\mathrm{x}$ & $\mathrm{x}$ & $\mathrm{x}$ & + & $1.28 \pm 0.02$ \\
\hline
\end{tabular}

Note: Data in bold indicate the most potential isolates attributed to PGP properties. HL = hypocotyl length; RL = radicular length; LR = the number of lateral roots; $\mathrm{WW}=$ wet weight; DW= dry weight. $\mathrm{v}=$ significantly promote growth parameter, $\mathrm{x}=$ not significantly promote growth parameter, $+=$ grow,$-=$ not grow 


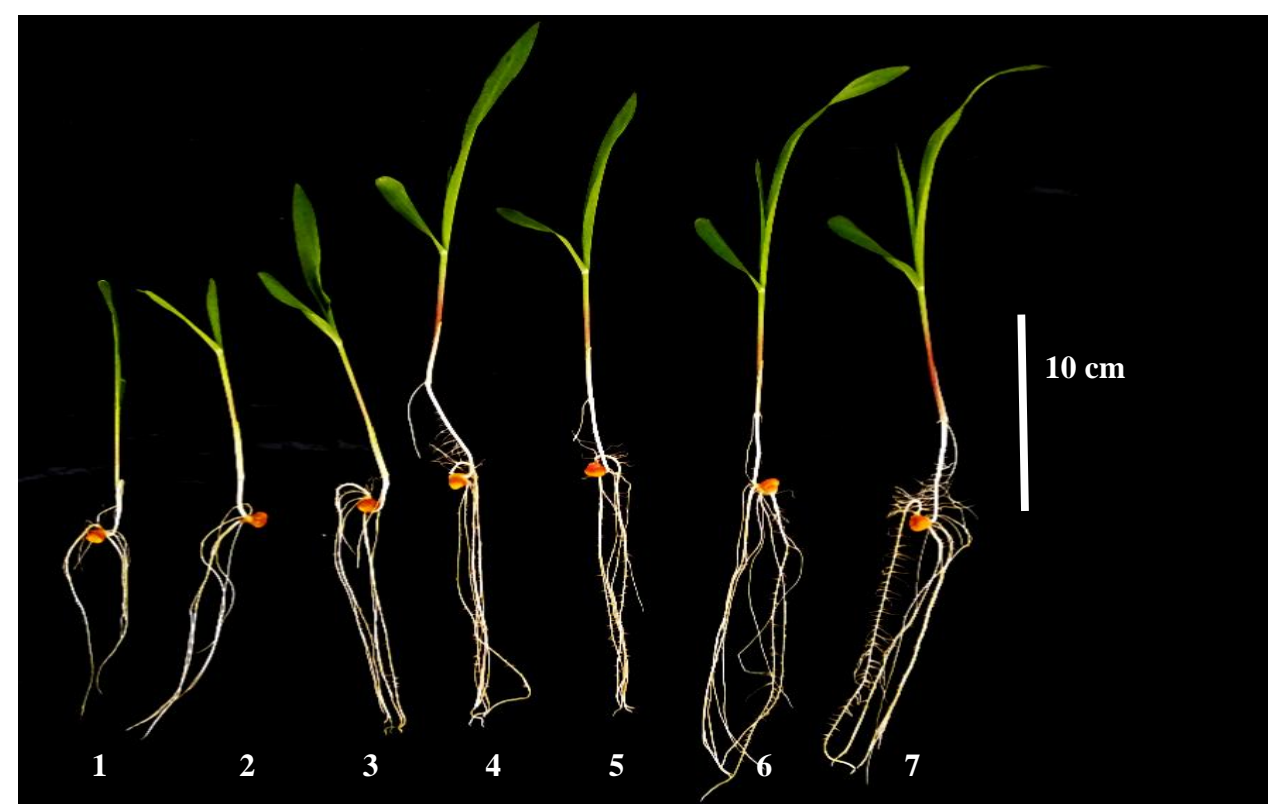

Figure 1. Growth response of maize sprouts inoculated on ISP4 medium (1), distilled water (2), ARJ 36 (3), ARJ 44 (4), ARJ 31 (5), ARJ 15 (6), ARJ 81 (7) after 7 days incubation at room temperature $\left( \pm 27^{\circ} \mathrm{C}\right)$

\section{IAA production of maize rhizosphere Actinomycetes}

Thirty Actinomycetes isolates $(100 \%)$ were able to produce IAA in different concentrations ranging from 1.05 to $26.89 \mathrm{ppm}$ (Table 1). They could be classified into three groups, classified as high, moderate, and low IAA producers isolate. Five isolates categorized as high IAA producers (19.26-26.89 ppm), 9 isolates were categorized as moderate IAA producers (9.07-16.3 ppm), and 16 isolates were categorized as low IAA producers (1.05-8.41 ppm). The highest IAA concentration was produced by ARJ 21 isolate $(26.89 \mathrm{ppm})$, whereas the lowest IAA concentration was exhibited by ARJ 12 isolate (1.05 ppm). Pseudomonas aeruginosa, served as a positive control, was able to produce IAA of $10.05 \mathrm{ppm}$, while Staphylococcus aureus, as a negative control, was unable to produce IAA.

\section{Plant growth-promoting (PGP) activities of maize rhizosphere Actinomycetes}

Twenty-eight isolates (93\%) of 30 isolates tested showed PGP activities and could stimulate different growth parameters of Pertiwi 3 hybrid maize sprouts (Figure 1). As shown in Table 2, nine isolates were able to significantly increase the five growth parameters, including the number of lateral roots, hypocotyl length, radicular length, dry weight, and wet weight. These isolates were ARJ 81, ARJ 51, ARJ 38, ARJ 14, ARJ 44, ARJ 32, ARJ 16, ARJ 28, and ARJ 15. Other Actinomycetes isolates were able to enhance only in particular growth parameters. Ten isolates (ARJ 61, ARJ 36, ARJ 47, ARJ 24, ARJ 27, ARJ 43, ARJ 23, ARJ 34, ARJ 49, and ARJ 13) were able to promote 4 parameters. Six isolates coded as ARJ 21 isolate, ARJ 11, ARJ 37, ARJ 22, ARJ 45, and ARJ 31, promoted three growth parameters. Two isolates, ARJ 48 and ARJ 42 were able to promote two growth parameters. One isolate, coded as ARJ 18, promoted only one parameter. Other 2 isolates, namely ARJ 12 and ARJ 17 were unable to enhance maize sprout growth. Their growth parameter values were not significantly different compared to the controls (Table 2).

\section{Actinomycetes growth in $\mathbf{N}$-free medium}

Thirty Actinomycetes isolates tested (100\%) were able to grow on nitrogen-free medium (Table 1). Based on the abundance and thickness of the colonies, $\mathrm{N}$-fixation activities of all isolates were found to be diverse.

\section{Phosphate solubilization properties of Actinomycetes}

Twenty-one isolates $(70 \%)$ of 30 isolate tested, were able to solubilize phosphate in Pikovskaya medium as indicated by clear zone formation around Actinomycetes colonies. Phosphate solubilization index of that isolates was found to be varies ranging from 1.09-1.89 (Table 1). ARJ 81 isolates exhibited the highest index $(1.89 \pm 0.25)$, while ARJ 49 showed the lowest index $(1.09 \pm 0.08)$.

\section{Maize rhizosphere Actinomycetes identity based on 16S-rRNA sequence}

The five Actinomycetes isolates (ARJ 15, ARJ 38, ARJ 44, ARJ 51, and ARJ 81), were screened based on their plant growth-promoting characters, were selected for molecular identification by using the PCR technique. In instance, they were capable in synthesizing IAA, enhancing maize sprouts growth in planta, solubilizing phosphate, growing on nitrogen-free medium, and negative hypersensitivity reactions. All 5 isolates were closely related to the genus Streptomyces and contained DNA homology between $99-100 \%$ in the 16S-rRNA sequences, yet distinct in the taxa of species and strains (Table 3). Consistently, these five isolates also located in the same clade in the phylogenetic tree (Figure 2). 
Table 2. Plant growth-promoting activities of 28 Actinomycetes isolates in promoting five growth parameters of maize sprouts after 7 days of incubation in room temperature $\left( \pm 27^{\circ} \mathrm{C}\right)$

\begin{tabular}{|c|c|c|c|c|c|c|}
\hline $\begin{array}{c}\text { Experimental } \\
\text { group }\end{array}$ & Treatment & $\begin{array}{c}\text { Hypocotyl length } \\
\text { (cm)* }\end{array}$ & $\begin{array}{c}\begin{array}{c}\text { Radicular length } \\
(\mathrm{cm}) *\end{array} \\
\end{array}$ & $\begin{array}{l}\text { The number of } \\
\text { lateral roots* }\end{array}$ & $\begin{array}{c}\text { Wet weight } \\
(\mathrm{g}) *\end{array}$ & $\begin{array}{c}\text { Dry weight } \\
(\mathrm{g}) *\end{array}$ \\
\hline \multirow[t]{12}{*}{1} & Distilled water & $9.60^{\mathrm{a}}$ & $12.85^{b}$ & $56.81^{\mathrm{a}}$ & $8.11^{\mathrm{a}}$ & $1.33^{\mathrm{a}}$ \\
\hline & Uninoculated ISP4 & $10.83^{b}$ & $12.55^{\mathrm{b}}$ & $59.37^{\mathrm{a}}$ & $8.40^{\mathrm{a}}$ & $1.23^{\mathrm{a}}$ \\
\hline & ARJ 44 & $13.23^{\mathrm{f}}$ & $14.47^{\mathrm{c}}$ & 119.78 de & $11.78^{\mathrm{de}}$ & $1.57 \mathrm{bcd}$ \\
\hline & ARJ 81 & $12.80^{\mathrm{de}}$ & $12.98^{\mathrm{c}}$ & $135.04^{\mathrm{e}}$ & $11.42^{\mathrm{e}}$ & $1.71^{\mathrm{e}}$ \\
\hline & ARJ 37 & $12.23^{\mathrm{de}}$ & $12.70^{\mathrm{b}}$ & $102.04^{\mathrm{cd}}$ & $10.34^{\mathrm{bcd}}$ & $1.32^{\mathrm{a}}$ \\
\hline & ARJ 28 & $12.23 \mathrm{de}$ & $15.55^{\mathrm{de}}$ & $118.1^{\mathrm{de}}$ & $12.17^{\mathrm{e}}$ & $1.59 \mathrm{bcd}$ \\
\hline & ARJ 45 & $12.21^{\mathrm{de}}$ & $14.96^{\mathrm{c}}$ & $59.85^{\mathrm{a}}$ & $9.01^{\mathrm{ab}}$ & $1.52^{\mathrm{bcd}}$ \\
\hline & ARJ 15 & $12.05^{\mathrm{cde}}$ & $16.30^{\mathrm{e}}$ & $97.93^{\mathrm{cd}}$ & $11.17^{\mathrm{cde}}$ & $1.61^{\text {cde }}$ \\
\hline & ARJ 31 & 12.03 cde & $14.46^{\mathrm{c}}$ & $70.37 \mathrm{ab}$ & $9.20^{\mathrm{ab}}$ & $1.52^{\mathrm{bcd}}$ \\
\hline & ARJ 61 & 11.82 cde & $11.70^{\mathrm{a}}$ & $136.74^{\mathrm{e}}$ & $12.67^{\mathrm{e}}$ & $1.46^{\mathrm{b}}$ \\
\hline & ARJ 36 & $11.61^{\mathrm{bcd}}$ & $15.44^{d}$ & $88.07^{b c}$ & $10.31 \mathrm{bcd}$ & $1.64 \mathrm{de}$ \\
\hline & ARJ 17 & $10.57^{\mathrm{b}}$ & $11.40^{\mathrm{a}}$ & $71.26^{a b}$ & $8.51^{\mathrm{a}}$ & $1.27^{\mathrm{a}}$ \\
\hline \multirow[t]{12}{*}{2} & Distilled water & $11.24^{b c}$ & $14.88^{\mathrm{a}}$ & $77.41^{\mathrm{a}}$ & $10.41^{\mathrm{ab}}$ & $1.21^{\mathrm{a}}$ \\
\hline & Uninoculated ISP4 & $11.15^{\mathrm{ab}}$ & $15.87^{\mathrm{a}}$ & $71.96^{\mathrm{a}}$ & $10.02^{\mathrm{a}}$ & $1.25^{\mathrm{ab}}$ \\
\hline & ARJ 21 & $12.04^{\mathrm{f}}$ & $15.80^{\mathrm{a}}$ & $121.96^{b c}$ & $12.58^{\mathrm{bcd}}$ & $1.65^{\mathrm{d}}$ \\
\hline & ARJ 47 & 11.79 ef & $19.02^{\mathrm{cd}}$ & $140.26^{b c}$ & $14.36^{\mathrm{de}}$ & $1.52^{\mathrm{bcd}}$ \\
\hline & ARJ 51 & $11.72 \mathrm{def}$ & $17.24^{\mathrm{b}}$ & $132.48^{b c}$ & $12.18^{\mathrm{cd}}$ & $1.66^{\mathrm{d}}$ \\
\hline & ARJ 24 & $11.72 \mathrm{def}$ & $21.92^{\mathrm{e}}$ & $132.41^{b c}$ & $15.61^{\mathrm{e}}$ & $1.43^{\mathrm{abcd}}$ \\
\hline & ARJ 11 & $11.69 \mathrm{def}$ & $17.65^{b c}$ & $120.81^{b c}$ & $11.48^{a b c}$ & $1.31 \mathrm{abc}$ \\
\hline & ARJ 27 & $11.66^{\text {def }}$ & $18.40^{\text {bcd }}$ & $116.56^{b}$ & $13.55^{\text {cde }}$ & $1.49^{\mathrm{abcd}}$ \\
\hline & ARJ 12 & $11.18^{a b}$ & $15.22^{\mathrm{a}}$ & $84.73^{\text {a }}$ & $10.40^{\mathrm{ab}}$ & $1.26^{\mathrm{ab}}$ \\
\hline & ARJ 43 & $11.33^{a b c}$ & $21.22^{\mathrm{e}}$ & $135.33 \mathrm{bc}$ & $15.41^{\mathrm{e}}$ & $1.53^{\mathrm{cd}}$ \\
\hline & ARJ 22 & $11.23^{b c}$ & $18.44^{\mathrm{bcd}}$ & $121.30^{b c}$ & $13.69^{\text {cde }}$ & $1.51^{\mathrm{bcd}}$ \\
\hline & ARJ 23 & $10.76^{\mathrm{a}}$ & $19.66^{d}$ & $152.74^{\mathrm{c}}$ & $15.02^{\mathrm{e}}$ & $1.55^{\mathrm{cd}}$ \\
\hline \multirow[t]{12}{*}{3} & Distilled water & $10.02^{a b}$ & $17.85^{\mathrm{a}}$ & $73.56^{\mathrm{a}}$ & $10.33^{\mathrm{a}}$ & $1.28^{\mathrm{ab}}$ \\
\hline & Uninoculated ISP4 & $9.92^{\mathrm{a}}$ & $17.74^{\mathrm{a}}$ & $81.11^{\mathrm{a}}$ & $10.75^{\mathrm{ab}}$ & $1.21^{\mathrm{a}}$ \\
\hline & ARJ 18 & $10.05^{b}$ & $18.81^{\mathrm{ab}}$ & $85.23^{\mathrm{b}}$ & $11.57^{\text {abcd }}$ & $1.38^{\mathrm{bc}}$ \\
\hline & ARJ 48 & $10.24 \mathrm{bc}$ & $18.56^{\mathrm{bc}}$ & $74.98^{b}$ & $11.94 \mathrm{bcd}$ & $1.33^{\mathrm{abc}}$ \\
\hline & ARJ 13 & $10.94^{\mathrm{cd}}$ & $21.01 \mathrm{de}$ & $168.52^{c}$ & $11.39 \mathrm{abc}$ & $1.45^{\text {cde }}$ \\
\hline & ARJ 34 & $11.13^{\mathrm{d}}$ & $21.55^{\mathrm{e}}$ & $181.44^{\mathrm{c}}$ & $12.50^{\mathrm{cd}}$ & $1.33^{\mathrm{abc}}$ \\
\hline & ARJ 16 & $11.48^{\mathrm{cd}}$ & $19.72^{\mathrm{bcd}}$ & $159.67^{b c}$ & $12.78^{\mathrm{cd}}$ & $1.58 \mathrm{de}$ \\
\hline & ARJ 14 & $11.04^{\mathrm{cd}}$ & $19.41 \mathrm{bc}$ & $155.59^{\mathrm{bc}}$ & $12.75^{\mathrm{cd}}$ & $1.48^{\text {cde }}$ \\
\hline & ARJ 38 & $11.01^{\mathrm{cd}}$ & $19.69 \mathrm{bcd}$ & $160.33^{b c}$ & $12.43^{\mathrm{cd}}$ & $1.63^{\mathrm{e}}$ \\
\hline & ARJ 32 & $11.09^{\mathrm{cd}}$ & $20.39^{\text {cde }}$ & $160.19^{b c}$ & $12.76^{\mathrm{cd}}$ & $1.66^{\mathrm{e}}$ \\
\hline & ARJ 42 & $11.17^{\mathrm{cd}}$ & $19.19^{a b c}$ & $157.81^{b c}$ & $11.69^{a b c d}$ & $1.31 \mathrm{abc}$ \\
\hline & ARJ 49 & $10.89^{\mathrm{cd}}$ & $21.35^{\mathrm{e}}$ & $161.44^{b c}$ & $12.48^{\mathrm{cd}}$ & $1.35^{\mathrm{abc}}$ \\
\hline
\end{tabular}

Note: *The data was performed as the average of three replications in which each replication consist of 9 maize sprouts. Data in the bold and different letter above the number indicate significant promotion of five growth parameters as well as compared to the control based on the Duncan test $(\alpha=0.05)$.

Table 3. Identity of 5 potential Actinomycetes isolates attributed to plant growth-promoting characters

\begin{tabular}{clccc}
\hline $\begin{array}{c}* \text { Isolate } \\
\text { Code }\end{array}$ & \multicolumn{1}{c}{$\begin{array}{c}\text { Closely identical strain } \\
\text { (Acc. Number) }\end{array}$} & $\begin{array}{c}\text { Query } \\
\text { Cover (\%) }\end{array}$ & $\begin{array}{c}\text { E-value } \\
\text { Similarity } \\
(\boldsymbol{\%})\end{array}$ \\
\hline & & & 100 & 0.0 \\
ARJ 15 & Streptomyces tendae strain M23 (HM594286.1) & 100 & 0.0 & 100 \\
ARJ 38 & Streptomyces collinus subsp. albescens strain NBRC12547 (AB184101.2) & 100 & 0.0 & 100 \\
ARJ 44 & Streptomyces sp. strain E2N108 (KX279552.1) & 100 & 0.0 & 99 \\
ARJ 51 & Streptomyces cellulosae strain JJ127 (KX352811.1) & 100 & 0.0 & 99 \\
ARJ 81 & Streptomyces sp. strain S14 (HG428657.1) & & \\
\hline
\end{tabular}

Note: *The sequences have been deposited into NCBI GenBank under the accession number MK240492.1-MK240496.1. 


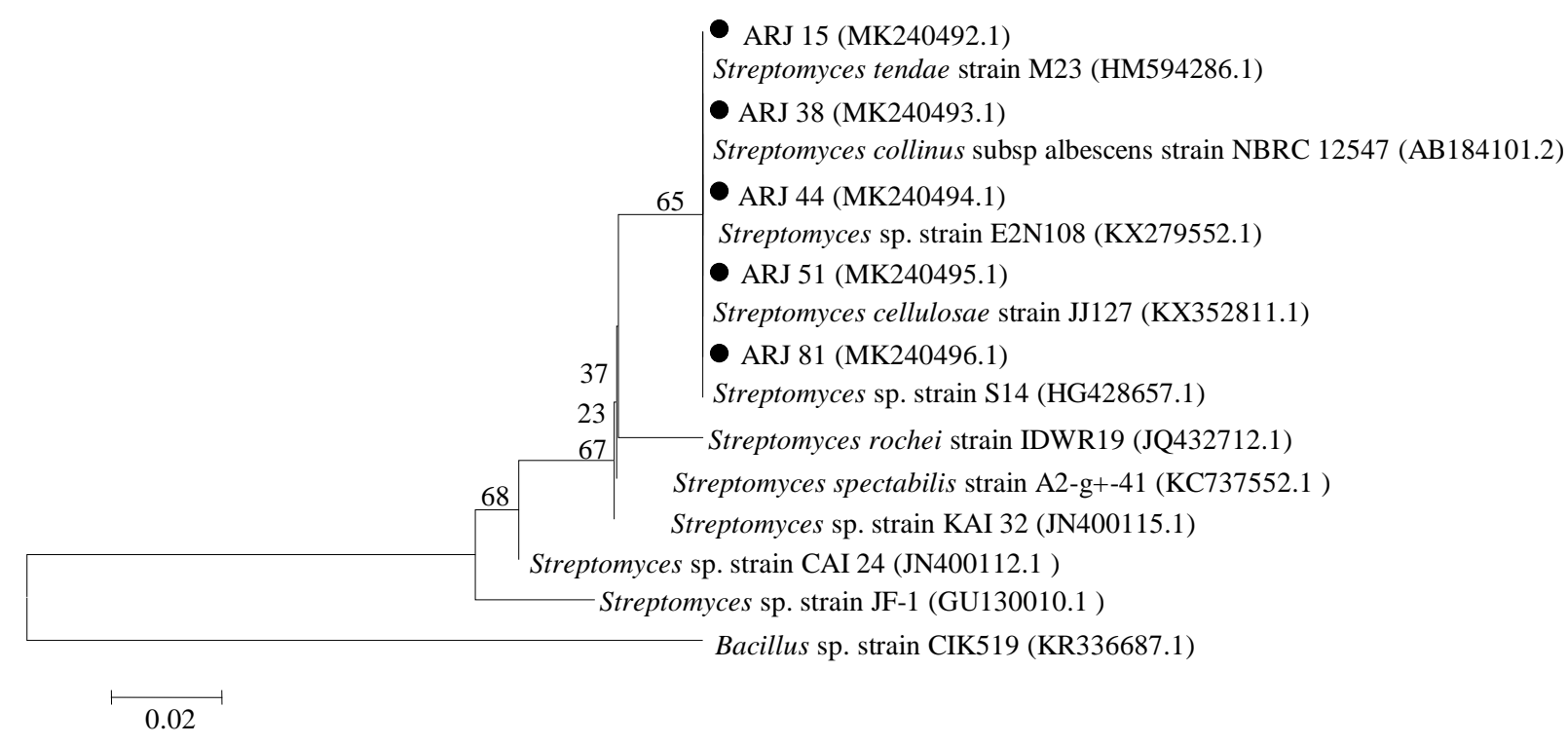

Figure 2. Phylogenetic tree of 5 Actinomycetes isolates attributed to plant growth-promoting properties compared to their closely identical strain based on 16S-rRNA sequences and constructed by using the neighbor-joining method with a bootstrap value of 1000x in MEGA 7.0 software

\section{Discussion}

Actinomycetes have been considered as one of the prominent group of soil microorganisms, and their types of Actinomycetes were greatly influenced by physical and chemical aspect including soil type, climatic condition, geographical location, and soil $\mathrm{pH}$ (Arifuzzaman et al. 2010). One potential source to collect Actinomycetes is rhizosphere. Secretion of root exudates in this habitat provides a high nutritional content needed for microbial growth. It is well known that more than $80 \%$ of the bacteria isolated from this habitat are capable of producing Indole3-acetic acid (IAA) (Spaepen and Vanderleyden 2011). In this study, 30 Actinomycetes isolates were isolated from maize rhizosphere collected from East Nusa Tenggara, Indonesia. Thirty collected isolates exhibited variations in their colony and cellular morphology. All isolates did not show pathogenicity to plant as indicated by no necrotic zone formed on tobacco leaves after being inoculated with Actinomycetes, and these isolates also have the potential to promote plant growth. Actinomycetes which potential as biofertilizer agents are expected to produce phytohormones, one of which is IAA. This hormone plays a significant role in stimulating and regulating plant cells development (Joshi and Bath 2011).

Interestingly, all thirty isolates $(100 \%)$ in this study were able to produce IAA in liquid ISP2 medium supplemented with L-tryptophan. IAA concentration produced by each isolate was found to be varies ranging from 1.05 to $26.89 \mathrm{ppm}$. The highest IAA concentration was $26.89 \mathrm{ppm}$ produced by ARJ 21 isolate, while the lowest IAA concentration was $1.05 \mathrm{ppm}$ produced by ARJ 12 isolates. Differences of IAA production could be influenced by the ability of Actinomycetes to convert L- tryptophan to IAA as well as the biochemical and genetic characters of each isolate (Spaepen and Vanderleyden 2011).

Similarly, in the previous study by Kaur et al. (2017) reported that 16 isolates of Actinomycetes isolated from the rhizosphere of Gladiolus were able to produce IAA with concentrations ranging from 8.1 to $79.9 \mathrm{ppm}$. Anwar et al. (2016) also reported that 30 Actinomycetes isolated from the rhizosphere of wheat and tomatoes were able to produce IAA with concentrations ranging from 10-79.5 $\mathrm{ppm}$. The results of this study indicated that the most Actinomycetes isolated from the rhizosphere of maize were able to produce IAA. It showed that maize rhizosphere is a potential source for searching biofertilizer candidates. This data also could be the microbiological evidence of mutualistic interaction between microbes and their activities in promoting plant growth.

The capability of Actinomycetes from rhizosphere in producing IAA was not in line with their plant growth promotion activities. Actinomycetes with high and low IAA production were able to enhance maize sprouts growth. It is presumably due to IAA produced by plants have been able to increase plant growth through cell division and elongation, so the additional IAA produced by microbes has no significant effect on plant growth (Pamungkas 2009). Increased growth was higher in maize sprouts inoculated with moderate IAA producers (8.4-16.6 ppm) and low IAA concentrations (1-8.3 ppm). The isolates coded as ARJ 11, ARJ 21, and ARJ 49 (high IAA producers) only promoted three growth parameters, while nine other isolates significantly enhanced 5 growth parameters. At high IAA concentrations, only a few isolates are capable of promoting growth in all five growth 
parameters. High IAA concentrations are likely to inhibit plant growth and trigger ethylene synthesis (Putrie et al. 2013). It is also influenced by the ability of isolates to colonize roots and defend themselves, as reported by Nelson (2004).

The ability of Actinomycetes isolates to enhance nutrient availability needs to be evaluated. Actinomycetes which have the potential to promote plant growth are tested for their ability in fixing nitrogen and solubilizing phosphate. All 30 isolates (100\%) were able to grow on nitrogen-free medium suggesting their ability in fixing nitrogen from other sources, such as nitrogen from the air. Besides, 21 Actinomycetes isolates (70\%) also able to solubilize phosphate as indicated by clear zone formation on Pikovskaya media. The ability of these isolates in solubilizing phosphate and fixing nitrogen are expected to increase nutrient availability in soil.

Five isolates coded as ARJ 81, ARJ 44, ARJ 38, ARJ 15 , and ARJ 51 showed promising PGP properties. These isolates were able to produce IAA, significantly promote 5 growth parameters, able to grow on $\mathrm{N}$-free medium, and solubilize phosphate. Based on the 16S-rRNA gene, all five isolates were highly similar to Streptomyces sp. There were many reports which established the ability of rhizospheric soil Streptomycetes to promote plant growth including in sorghum and rice (Gopalakrishnan et al. 2013), banana (Chen at al. 2018), and Chickpea (Sreevidya et al. 2016). Surprisingly, these 5 isolates were located in the same clade in the phylogenetic tree, meaning that these isolates were closely similar according to $16 \mathrm{~S}-\mathrm{rRNA}$. Although 16S-rRNA gene is sufficient for bioinformatics analysis, however, its particular DNA sequence is not reliable for intra-strain differentiations.

Consequently, further study is necessary to confirm the identity of that isolates. The findings from this present study indicate the possibilities of the use of five maize rhizospheric Streptomyces spp. as biofertilizer for nutrient mobilization and maize growth promotion.

\section{ACKNOWLEDGEMENTS}

This work was funded by the Ministry of Research, Technology, and Higher Education of Indonesia through "Penelitian Dasar-Unggulan Perguruan Tinggi" (PD-UPT) 2018 [Contract No.: 129/SP2H/PTNBH/DRPM/2018] and 2019 [Contract No.: 3/E1/KP.PTNBH/2019] awarded to Aris Tri Wahyudi. Therefore, the authors thank for all the supports of this research.

\section{REFERENCES}

Anwar S, Ali B, Sajid I. 2016. Screening of rhizospheric actinomycetes for various in-vitro and in-vivo plant growth promoting (PGP) traits and for agroactive compounds. Front Microbiol 7: 1334.

Arifuzzaman M, Khatun MR, Rahman H. 2010. Isolation and screening of actinomycetes from Sundarbans soil for antibacterial activity. Afr J Biotechnol 9: 4615-4619.

Chen Y, Zhou D, Qi D, Gao Z, Xie J, Luo Y. 2018. Growth promotion and disease suppression ability of a Streptomyces sp. CB-75 from banana Rhizosphere soil. Front Microbiol 8 (2704): 1-18.

Gopalakrishnan S, Srinivas V, Sreevidya M, Rathore A. 2013. Plant growth-promoting activities of Streptomyces spp. in sorghum and rice. Springer Plus 2 (574): 1-8.

Gopalakrishnan S, Vadlamudi S, Bandikinda P, Sathya A, Vijayabharathi R, Rupela O, Kudapa H, Katta K, Varshney RK. 2014. Evaluation of Streptomyces strains isolated from herbal vermicompost for their plant growth-promotion traits in rice?. Microb Res 169 (1): 40-48.

Jeon LS, Kim H, Ahn T, Song H. 2003. Plant growth promotion in soil by some inoculated microorganism. J Microbiol 41 (4): 27-276.

Joshi P, Bath AB. 2011. Diversity and function of plant growth-promoting rhizobacteria associated with wheat rhizosphere in North Himalaya Region. Int J Environ Sci 16: 1135-1143.

Kaur J, Gangwar M, Kaur S. 2017. Screening of endophytic and rhizospheric actinomycetes with potential application for biocontrol of Fusarium wilt of Gladiolus. Int J Curr Microbiol App Sci 6 (7): 1345-1355.

Kresnatita S, Koesriharti, Santoso M. 2013. Pengaruh rabuk organik terhadap pertumbuhan dan hasil tanaman jagung manis. Indonesian Green Technology Journal 2 (1): 8-17. [Indonesian]

Martina K, Jan K, Tamas F, Ladislav C, Marek O, Genevieve LG. 2008. Development of a $16 \mathrm{~S}$ rRNA gene-based prototype microarray for the detection of selected actinomycetes genera. Anton Van Leeuw 94: 439-453.

Marques APGC, Pires C, Moreira H, Rangel AOSS, Castro PML. 2010. Assessment of the plant growth promotion abilities of six bacterial isolates using Zea mays as indicator plant. Soil Biol Biochem 42: 1229-1235.

Nelson LM. 2004. Plant growth-promoting rhizobacteria (PGPR): Prospects for new inoculants. Crop Manag 3 (1): 1-7.

Pamungkas FT, Darmanti S, Raharjo B. 2009. Pengaruh konsentrasi dan lama perendaman dalam supernatan kultur Bacillus sp.2 DUCC-BRKI.3 terhadap pertumbuhan stek horisontal batang jarak pagar (Jatropha curcas L.). J Sci Math 17 (3): 131-140. [Indonesian]

Patil HJ, Srivastava AK, Kumar S, Caudhari BL, Arora DK. 2010. Selective isolation, evaluation and characterization of antagonistic actinomycetes against Rhizoctonia solani. World J Microbiol Biotechnol 26: 2163-2170.

Putrie RFW, Wahyudi AT, Nawangsih AA, Husen E. 2013. Screening of rhizobacteria for plant growth-promoting and their tolerance to drought stress. Microbiol Indones 7 (3): 94-104.

Savci S. 2012. An agricultural pollutant: Chemical fertilizer. Int J Environ Sci Develop 3 (1): 77-80.

Spaepen S, Vanderleyden J. 2011. Auxin and plant-microbe interactions. Cold Spring Harbor Perspect in Biol 3 (4): 1-13.

Sreevidya M, Gopalakrishman S, Kudapa H, Varhsney RK. 2016. Exploring plant growth-promoting actinomycetes from vermicompost and rhizosphere soil for yield enhancement in chickpea. J Microbiol 47: 85-95.

Viaene T, Langendries S, Bernick S, Maes M. 2016. Streptomyces as Plants's best friend? FEMS Microbiol Ecol 92 (8): 1-10.

Wiraswati SM, Rusmana I, Nawangsih AA, Wahyudi AT. 2019. Antifungal activities of bacteria producing bioactive compounds isolated from rice phyllosphere against Pyricularia oryzae. J Plant Protect Res 59 (1): 86-94. 